\title{
Expanding the domain of the understandable in psychiatric illness: an updating of the Jasperian framework of explanation and understanding
}

\author{
K. S. Kendler ${ }^{1 *}$ and J. Campbel1 ${ }^{2}$ \\ ${ }^{1}$ Virginia Institute of Psychiatric and Behavioral Genetics, and Departments of Psychiatry, and Human and Molecular Genetics, Virginia \\ Commonwealth University School of Medicine, Richmond, VA, USA \\ ${ }^{2}$ Department of Philosophy, University of California, Berkeley, CA, USA
}

Jaspers famously argued that psychiatry has two distinct ways of knowing about psychiatric illness: explanation which utilizes natural sciences, and objective and empirical methods; and understanding-which reflects our subjective, empathic appreciation of our patients' experiences. He saw a clear division between these two approaches and considered a number of psychotic symptoms to be fundamentally un-understandable. We here argue for an updating of Jasper's view that includes the possibility, due to advances in neuroscience and neuropsychology, for explanation-aided understanding. We describe two scientific explanatory models for kinds of psychotic symptoms deemed un-understandable by Jaspers that illustrate this process. The first describes how dysfunction of the brain dopamine salience system may produce ideas of reference. The second demonstrates that symptoms of made-actions may be caused by abnormalities in the feed-forward motor control system. Both of these models begin at a biological level describing pathological brain processes in neuroscience language but then utilize neuropsychology to 'translate' from brain dysfunction into the mental. Both models break down the pathological pathways into individual subjective elements (particularly misattributing meaning to environmental stimuli and having body parts moved by others) that are understandable because they are common to nearly all of us. The neuropsychology provides hypotheses about the functional and physiological structures underlying familiar subjective experiences. Analyses of disorders of those structures thus provide a scaffold for expanding our empathic understanding of the subjective experiences of those suffering from psychiatric illness.

Received 25 September 2012; Revised 8 December 2012; Accepted 11 December 2012; First published online 24 January 2013

Key words: Explanation, Jaspers, neuropsychology, philosophy, understanding.

\section{Introduction}

In his deservedly famous book, General Psychopathology, Karl Jaspers, building on the prior work of Wilhelm Dilthey (Wikipedia contributors, 2010), argued that psychiatry has two distinct ways of knowing about mental illness (Jaspers, 1963). One is shared with the natural sciences and seeks to establish causal relationships using objective empirical methods. This approach Jaspers termed 'explanation'. The second is unique to the human sciences and reflects the subjective, empathic appreciation of the experiences of our patients. This approach Jaspers termed 'understanding'. For Jaspers, explanation was an effortful process involving hypothesis generation, data collection and

\footnotetext{
* Address for correspondence: K. S. Kendler, M.D., Virginia Institute for Psychiatric and Behavioral Genetics, Virginia Commonwealth University Medical School, Box 980126, 800 E. Leigh Street, Room 1-123, Richmond, VA 23298-0126, USA.

(Email: kendler@hsc.vcu.edu)
}

interpretation. By contrast, understanding was immediate and 'self-evident' as reflected 'the inner, subjective, direct grasp of psychic connectedness' (Jaspers, 1963, p. 307). In an earlier essay, Jaspers succinctly summarizes the difference between these two ways of knowing (in this and other quotes of Jaspers, italics are in the original): 'We understand an action from motives; we explain a movement causally by nerve stimulation' (Jaspers, 2007, p. 174).

This is a central point and worth emphasizing with two short examples. When you see a friend strike his shin against a table leg, wince, hop up and down, rubbing his leg and cursing under his breath, you are immediately able to empathize with the pain he is experiencing. No calculations or confidence intervals are needed. When you see a depressed patient for a first evaluation and she begins, with tender lovingness, to describe the depth of her feelings for her husband of 18 years, of the warmth of her recollections about raising their three children together and of her hurt at discovering his unfaithfulness, at which point she 
begins to cry, you can directly and without effort feel her anguish.

Explanation, by contrast, reflects the 'objective demonstration of connections, effects and ruling principles, which cannot be understood by empathy and are only explicable in terms of cause and effect' (Jaspers, 1963, p. 307). He summarizes this difference as follows:

In the natural sciences, we find causal connections only but in psychology our bent for knowledge is satisfied with the comprehension of quite a different sort of connection (Jaspers, 1963, p. 302).

That is, while explanation has wide applicability as a way of knowing - it could be used for everything from simple chemical systems to galaxies understanding is restricted to conscious intentional beings. When dealing with our fellow humans, we are not innately satisfied with only a causal explanation of their behavior. We want and need to understand.

But, as Jaspers notes, understanding has its problems: 'There is no limit to the discovery of causes [i.e. explanation] ... with understanding, there are limits everywhere' (Jaspers, 1963, p. 305) and 'We now know that only certain types of the psychic are accessible to our understanding' (Jaspers, 2007, p. 180). We share with Jaspers an interest in one area in which the process of understanding appears to hit a brick wall. These are a particular set of psychotic phenomena that he terms 'un-understandable'. Regarding what he termed primary delusions, Jaspers wrote:

If we try to get some closer understanding of these primary experiences of delusions, we soon find we cannot really appreciate these quite alien modes of experience. They remain largely incomprehensible, unreal and beyond our understanding (Jaspers, 1963, p. 98).

Elsewhere he considers such delusions 'psychologically irreducible' (Jaspers, 1963, p. 96). Jaspers goes on to describe a range of un-understandable psychotic symptoms. We focus on two symptom groups. The first is concerned with abnormalities of meaning and significance. He writes:

The experiences of primary delusion are analogous to ... seeing of meaning.... There is an immediate, intrusive knowledge of the meaning and it is this which is itself the delusional experience. ... Significance appears unaccountably, suddenly intruding into the psychic life (Jaspers, 1963, pp. 99, 103).

He gives several examples of psychotic cases with extensive delusions of reference illustrating the apparent eruption into consciousness of new meanings associated with their observations of the world around them. In his classic monograph on schizophrenia, Bleuler provides several clear examples, 'Everybody is in the street because of him; each gesture of these people has some significance for him; newspaper advertisements refer to him' (Bleuler, 1950, p. 133).

The second un-understandable phenomena are psychotic passivity experiences later considered one of the Schneiderian symptoms of schizophrenia (Mellor, 1970).

[The patient] does not feel master of his own thoughts ... he feels in the power of some incomprehensible external force.... The thought arises and with it a direct awareness that it is not the patient but some external agent that thinks it. [The patient feels] ... an elementary experience of being actually influenced (Jaspers, 1963, pp. 122-123).

Bleuler also provides an excellent description:

Action is split off from the conscious personality of the patient. The limbs do something, the lips say something, of which the patient is informed by his senses as if he were an observer during his action, as if he were a third person (Bleuler, 1950, p. 201).

These sorts of primary delusional experiences, Jaspers considered to be un-understandable.

\section{Expanding the boundaries of the understandable}

Developments in neuroscience and neuropsychology can, we contend, now permit us to expand the boundaries of the understandable in psychiatric illness. In Jasperian terms, we call this process explanationaided understanding. Many times in the past, scientific developments have permitted humans to expand our experiences of our universe. The microscope and the telescope have allowed us to peer into previously unknown worlds. While the mind-brain problem poses subtler issues than those of the optics of seeing things very small or far away, nonetheless there are parallels. Neuroscience, in general, and the functional analysis of the brain through neuropsychology, in particular, can allow us to understand empathically aspects of human experience previously beyond our grasp.

What Jaspers called the 'immediacy' of understanding should not be interpreted as to exclude the relevance of empirical research to understanding. Empathic understanding of another may require insight into a whole host of factors, including, for example, historical processes, economic context, social hierarchies, and so on. The present point is that neuropsychology provides another such dimension for the grounding of understanding.

We provide two illustrations of this process that address the key examples of un-understandability discussed by Jaspers: intrusion of inappropriate meaning into consciousness leading to ideas and delusions of reference; and the emergence of 'made' actions. The force of our argument does not depend 
upon the veracity of the specific scientific theories here reviewed. We present them as plausible examples of the kinds of explanations that we will discover as we increasingly clarify the nature of the brain-mind dysfunctions underlying psychotic symptomatology.

Both of these explanations begin at a biological level that describes pathological brain processes in neuroscience language. But they then both add a neuropsychological component which 'translates' the underlying brain dysfunction into the realm of the mental. It is here - with a functional definition of what goes wrong with the brain and our associated capacity to sense what that might feel like - that we can connect explanation and understanding.

A wide range of research in rodents and primates indicates that midbrain dopamine (DA) neurons not only play a critical role in reward and positive motivation, but also provide signals which 'tag' other forms of salient experiences including aversive and alerting events. One class of DA neurons reflects the motivational value of stimuli and others, of particular interest to us, encode motivational salience. As summarized in a recent review:

Both types of dopamine neurons are augmented by an alerting signal involved in rapid detection of potentially important sensory cues. We hypothesize that these dopaminergic pathways for value, salience, and alerting cooperate to support adaptive behavior... Alerting signals sent to motivational salience coding DA neurons would support orienting of attention to the alerting stimulus, engagement of cognitive resources to discover its meaning and decide on a plan for action, and increase motivation levels to implement this plan efficiently (Bromberg-Martin et al. 2010, pp. 815, 817; italics added).

As articulated by Kapur in an incisive earlier review:

Dopamine mediates the conversion of the neural representation of an external stimulus from a neutral and cold bit of information into an attractive or aversive entity. In particular, the mesolimbic dopamine system is seen as a critical component in the 'attribution of salience,' a process whereby events and thoughts come to grab attention, drive action, and influence goal-directed behavior because of their association with reward or punishment (Kapur, 2003, p. 14).

In simpler terms, firings of these DA neurons that encode motivational salience provide a signal to the organism saying: 'Pay attention to this stimulus. It is important. Figure out what is going on!'

What would we expect to happen if these DA salience neurons fired at inappropriate times? As suggested by Kapur and others, the result would be the incongruous intrusion of meaning into consciousness. This meaning-carrying with it a sense of significance - would be associated with whatever the individual was then observing in the environment.
It then takes only a small step to imagine that an idea of reference could thereby be produced.

This is precisely the process that Jaspers considered foundational to one form of un-understandable experiences. To be concrete, patient $X$ is watching the nightly news. His DA salience neurons misfire. He has the sense of some immediate meaning and importance in the commentator's comments. He seeks to 'discover the meaning' of the event and realizes that the commentator really is looking at him, and notices that his newscast may contain hidden messages to him. It takes little imagination to realize how easily a delusion of reference might emerge from this primary experience.

Assume we were at some time in the future when it has been well established that the DA salience system is dysfunctional in schizophrenia. You are interviewing $\mathrm{Mr} \mathrm{X}$ as he describes this experience to you. Will you, as Jaspers suggests, find this entirely ununderstandable, as a 'quite alien mode of experience' ? Or, in the context of your knowledge of the pathophysiology of schizophrenia, will this be understandable? Will you say to yourself, 'Oh, of course, another misfiring of his salience system?' We suggest that the latter will be more likely. Given our expanded knowledge of neuroscience, we can convert these experiences of the intrusion of meaning into consciousness from the un-understandable to the understandable.

What makes it so easy to empathize with the story of $\mathrm{Mr} X$ ? In normal life, we have all experienced a misattribution of meaning to an environmental stimulus. For example, you are waiting at an airport for a loved one. You see, in a crowd of passengers, a person in the distance who seems to be her. You jump to your feet and, with a rush of emotion, move toward her to embrace. As you get closer, you realize you were wrong. There was some similarity in body size, gait or clothing that caused you to think this person was your loved one. Your emotional response was misattributed. Through an error in your perceptual system, meaning was placed where it did not belong.

We can apply this salience model to a related psychotic phenomenon that Jaspers also considered ununderstandable: delusional atmosphere. He describes this clinical state [closely related to 'delusional mood' as described by Klaus Conrad (Mishara, 2010)] with this extract from a patient:

'Something is going on. Do tell me what on earth is going on?' ... Patients feel uncanny and that there is something suspicious afoot. Everything gets a new meaning.... Something seems in the air which the patient cannot account for, a distrustful, uncomfortable, uncanny tension ... Patients obviously suffer terribly under it and to reach some definite 
idea at least is like being relieved from some enormous burden (Jaspers, 1963, p. 98)

What would it be like if your DA salience system was chronically in overdrive? Everything around you, even the most mundane, would be drenched in new meaning. This might call to mind a common experience, perfected by film directors:

You are watching a movie, which opens by panning slowly over a peaceful suburban setting, for so long that you start to suspect that something is going wrong. Suddenly the movie soundtrack goes into overdrive, high staccato violins, and your eyes race over the screen. Your hands clench on your theatre seat. Here you are not being directed to look for any particular kind of meaning but something bad is about to happen soon but you do not know when or exactly where. Potential significance is everywhere.

Our second example concerns 'made-actions', in which patients experience their motor behavior as being forced upon them. This is one of Schneider's 'first rank' symptoms of schizophrenia (Schneider, 1959) and was described by Jaspers as 'an elementary experience of being actually influenced'. As a patient of Mellors' describes it, 'My fingers pick up the pen, but I don't control them. What they do is nothing to do with me' (Mellor, 1970). Blakemore and Frith, in a series of articles, have proposed a plausible neuropsychological model for this symptom (Frith et al. 2000; Blakemore, 2003; Blakemore et al. 2003) based on the 'feed forward model of motor control' (Miall et al. 1993).

In a simplified version, when the brain issues a motor command, it also sends an efferent copy of that command to a 'monitoring' center. That efferent copy includes an expected set of sensory consequences from the movement. In healthy functioning, the monitoring center compares the actual motor movement as it progresses to the planned efferent copy. If there are discrepancies, it makes mid-course corrections.

Now, imagine the following experiment. Sit in a chair with your right elbow resting on the armrest. Bring your arm from horizontal to a $45^{\circ}$ angle and then put it down again. Note your subjective sense of that movement. Then have a friend lift your arm in exactly the same way and return it. The subjective feeling is quite different. When you lifted the arm, it was 'your' movement. When your friend lifted your arm, that sense was lacking. Your arm was being moved, but not by you.

What is happening during these experiences in your forward output model of motor control? To anthropomorphize, in the first instance, when your arm is moving, a control monitor yells out, 'Is this one of ours?' Someone looks it up in the efferent copy bin and sure enough there is a copy of the 'lift your arm' command and the message goes back, 'One of ours!' So you have the subjective sense of it being your movement. In the second instance, no copy of the command is found in the efferent copy bin so the response comes back, 'Not one of ours!' You sense someone else is moving your arm.

As Blakemore theorizes, in schizophrenia, perhaps because of poor coordination between cortical areas, the efferent copy of a motor command is not always transferred to the monitoring center. As a result, when the patient makes a volitional motor movement, the coordination center looks for an efferent copy and cannot find one. The judgment, 'Not one of ours' comes back to the subject. The subject senses a 'madeaction'.

Again, assume we are in the future when this model has been confirmed by advanced neuroscience methods. You are interviewing Ms Y who, early in a schizophrenia psychosis, reports extensive madeactions, in which she feels others have 'taken over' her body and made her do things. Will you confront an un-understandable symptom beyond your empathic abilities? Or having learned about this symptom and tried out for yourself the arm-lifting exercises outlined above, can you understand what Ms Y's experiences must be like?

We chose to explicate ideas of reference and made-actions because they reflected paradigmatic examples of Jasper's concept of un-understandability. Interestingly, the empathic mechanisms at work in these two examples may differ. In trying to grasp how DA salience can produce ideas of reference, there is a rather immediate 'ah-ha' sense-because prior experiences of misattributing meaning to stimuli in our environment are so common for most of us. The made-action example takes more effort. Here, our ability to gain empathy results from breaking down the possible etiologic process into a series of steps, mentally reassembling them and then practising, at least in our mind's eye, to get the sense 'Oh, yes that must be what it would feel like.'

Functional neuropsychological models are also being developed for many psychiatric syndromes that can increase their understandability. We can potentially empathize with the repellant cruelty of sociopaths when its origin is explained as a brain-based developmental deficit in emotional learning that markedly blunts their ability to feel the pain they inflict on others (Blair, 2003; Anderson \& Kiehl, 2012). Neuropsychological models of impulsivity, based on deficits in inhibitory frontal pathways that result in inadequate 'top-down' behavioral control, may help us understand the risky and impulsive behaviors associated with attention deficit hyperactivity disorder (Robbins et al. 2012). 


\section{Context and limitations}

Neuroscience is not the only way of making symptoms understandable, which at first might seem bizarre and un-understandable. Indeed, Jaspers articulates two major categories of delusions:

One group emerges understandably from preceding affects, from shattering, mortifying, guilt-provoking or other such experiences, from false-perception. ... The other group is for us psychologically irreducible; phenomenologically it is something final (Jaspers, 1963, p. 96).

The first group, where the delusional beliefs arise understandably, Jaspers calls 'delusion-like ideas'. He saves the term 'delusions proper' for the ununderstandable, irreducible delusions. The concept of mood-congruent delusions in the Diagnostic and Statistical Manual of Mental Disorders (DSM), Third Edition (DSM-III), Third Edition Revised (DSM-III-R) and Fourth Edition (DSM-IV) is a direct intellectual descendent of Jaspers' concept of delusion-like ideas. A less recognized form of a delusion-like idea can emerge from hallucinations. Here is an example:

A young woman presented to the ER with a $24 \mathrm{~h}$ history of 'bizarre hallucinations and delusions.' After a nasty fight with her boyfriend, she experienced voices commenting on her behavior. As she walked from room to room within her apartment, even into the bathroom, the voices continued their commentary, noting what she was doing and often criticizing these actions ('Scrub those potatoes more carefully. They still have dirt on them.') She was deeply puzzled as to how 'they' could know what she was doing no matter where she went. Then it occurred to her that the electrician had been in the apartment last week to repair a faulty switch. Clearly, he must have installed some monitoring devices. She got a ladder from the basement and went through all the light fixtures, looking for cameras. She couldn't find any but became convinced that they must have been installed in places she could not get to.

Initially, the idea of cameras installed within light fixtures seems bizarre and un-understandable. However, taking a careful history and reconstructing her line of thought, it becomes possible to see that, given the content of the auditory hallucinations, these beliefs were neither bizarre nor irrational but rather an attempt to explain how the voices could always know what she was doing regardless of where she was in her apartment.

We are suggesting that neuroscience advances translated in neuropsychological models will expand the range of the understandable beyond that of careful history taking illustrated here. We cannot now define the likely limits of this effort. Functional/ neuropsychological explanations of psychiatric symptoms can provide the opportunity for, but do not guarantee, understandability. A key problem is that understandability is limited by the range of our own experiences and our own empathic imagination. While we have emphasized here a 'common sense' model of empathy, there are surely individual differences in what we see as understandable.

We have two tentative suggestions about the boundaries of explanation-enhanced understanding. First, it will not work when there is no underlying mental state with which to identify. If there is 'nothing that it is like to have the chorea-form movements of Huntington's chorea' - no identifiable subjective experience, perhaps because the pathology occurs in brain processes that do not enter consciousness - then no account of the neuropsychology of the disease, no matter how detailed, will aid our understanding. Second, the experience has to be within our intuitive grasp. If we were provided with a detailed functional explanation of the bat sonar perception system, would that help us understand what it is like to be a bat? (Nagel, 1979). Along with Thomas Nagel, we think not. We do not possess the elemental components necessary to put together an empathic link to understand what it is like to fly about at night catching insects through sonar waves set off by our screams and bouncing off our insect prey. The neuropsychological expansion of understanding relies on more than the functional analysis of brain functioning that is available in the case of the bat. It relies on the fact that neuropsychology provides hypotheses about the functional and physiological correlates of familiar subjective experiences, and thus puts us in a position to understand the significance of disturbances in those underlying structures.

Should we be hesitant about the ability of neuropsychology to expand the range of empathic understanding because this would demand that the mind-body problem be solved, that we now understand, at a fundamental level, how the brain produces the mind? No. Rather, the account demonstrates how the expansion of empathy through neuropsychology can be accomplished without resolution of this hardest part of the mind-body problem. We are relying merely on the fact that in certain cases, we have wellgrounded hypotheses about what structures underlie particular subjective experiences, even though we do not yet understand how it happens that those subjective correlates are generated. Neuropsychology does not of itself allow us to understand the mechanisms by which those brain states can realize a subjective life in the first place. In fact, at the moment, we do not understand at the most basic level how the mechanisms might work through which particular patterns of brain activity generate particular patterns of experience. Neuropsychology nonetheless does put us in a position to clarify which patterns of brain activity are generating which patterns of experience. 


\section{Implications for etiologic models for psychiatric illness}

Finally, this discussion can help us reflect on what we want to know about the etiology of psychiatric illness (Kendler, 2008). This point, too, can be best illustrated by a story. In the future, some research group finds a strong, incontrovertible, causal relationship between a particular set of DNA variants and risk for psychiatric disorder $X$. The lead scientist claims that, 'Now we have clarified the etiology of disorder $X$. ' This is clearly unsatisfactory because all that has been demonstrated is the start of a causal chain. This path would look something like this:

$$
\begin{aligned}
\text { Causal genetic variants } & \rightarrow \rightarrow \overrightarrow{\text { Disorder } \mathrm{X}} \rightarrow \rightarrow \rightarrow \rightarrow
\end{aligned}
$$

The middle is blank. While a specific network of genes was discovered, which if disordered leads to a high probability to disorder $X$, none of the processes that lie between the genes, on the one hand, and the illness, on another, have been clarified.

However, the lead scientist takes this criticism very seriously. She launches a major research program to track down the causal pathways. How would she do that? Would it be good enough if her team showed, in fine detail, how the pathogenic genetic variant leads to key changes in functioning and microanatomy of neurotransmitter systems $\mathrm{A}, \mathrm{B}$ and $\mathrm{C}$ that then make an impact on broader networks D, E and F? This causal chain might look like this:

$$
\begin{aligned}
\text { Causal genetic variants } & \rightarrow \mathrm{A} \rightarrow \mathrm{B} \rightarrow \mathrm{C} \rightarrow \mathrm{D} \\
& \rightarrow \mathrm{E} \rightarrow \mathrm{F} \rightarrow \text { Disorder } \mathrm{X}
\end{aligned}
$$

This is still not adequate as key links in the chain are missing. How do we get from this brain-based set of findings-changes in neurotransmitter systems A, B and $\mathrm{C}$ - to the symptoms of disorder $\mathrm{X}$ ? We need to do a functionalist analysis of the A to $\mathrm{F}$ pathway. What does this pathway do in the mind-brain system? What functions does it serve? If it is disordered, what would we expect to have happen? What symptoms would then emerge?

In this functional analysis, which will undoubtedly use neuropsychological box and arrow kinds of constructs, the impact of the changes that occur in the A to F biological pathway will be eventually 'translated' from the level of brain to the level of mind. The research group will likely iterate back and forth from a biological and functional view of the deficits they are uncovering (Kendler, 2008).

In Jasper's terms, a complete clarification of the etiology of psychiatric illness cannot be achieved entirely via biological, causal explanation. A functional level of analysis is required and it is these models that provide the rubric for developing an understanding of the experiences of illness. We have to understand which structures underpin the subjective experiences characteristic of the disorder. As argued previously (Kendler, 2005, 2012), we need a pluralist approach toward clarifying the etiology of psychiatric illness.

\section{Conclusions}

Jaspers could not foresee the potential illuminating power of modern neuroscience. While his insight into the distinction in psychiatry between explanation and understanding is foundational, his assumptions - that the two pathways to knowledge are unrelated and that the boundaries of the un-understandable are fixedmay have been in error. In this essay, we advocate for the concept of explanation-aided understanding. Neuropsychology can provide hypotheses about which subjective experiences are grounded in which functional or physiological structures, in both the pathological and non-pathological cases. Through analysis of disorders in the underlying functional or physiological structures, neuropsychology can provide a scaffold for expanding our empathic understanding of the subjective experiences of those suffering from psychiatric illness. This effort also clarifies for us what we seek for in a complete description of the etiology of psychiatric illness.

\section{Acknowledgements}

Peter Zachar Ph.D. provided helpful comments on an earlier version of this essay.

\section{Declaration of Interest}

None.

\section{References}

Anderson NE, Kiehl KA (2012). The psychopath magnetized: insights from brain imaging. Trends in Cognitive Sciences 16, 52-60.

Blair RJ (2003). Neurobiological basis of psychopathy. British Journal of Psychiatry 182, 5-7.

Blakemore SJ (2003). Deluding the motor system. Consciousness and Cognition 12, 647-655.

Blakemore SJ, Oakley DA, Frith CD (2003). Delusions of alien control in the normal brain. Neuropsychologia 41, 1058-1067.

Bleuler E (1950). Dementia Praecox, or The Group of Schizophrenias. International Universities Press: New York.

Bromberg-Martin ES, Matsumoto M, Hikosaka O (2010). Dopamine in motivational control: rewarding, aversive, and alerting. Neuron 68, 815-834. 
Frith CD, Blakemore S, Wolpert DM (2000). Explaining the symptoms of schizophrenia: abnormalities in the awareness of action. Brain Research and Brain Research Review 31, 357-363.

Jaspers K (1963). General Psychopathology. University of Chicago Press: Chicago.

Jaspers K (2007). Causal and 'understandable' relationships between events and psychosis in dementia praecox (schizophrenia). In Anthology of German Psychiatric Texts (ed. Henning Sass), pp. 174-294. Blackwell Publishing: Oxford.

Kapur S (2003). Psychosis as a state of aberrant salience: a framework linking biology, phenomenology, and pharmacology in schizophrenia. American Journal of Psychiatry 160, 13-23.

Kendler KS (2005). Toward a philosophical structure for psychiatry. American Journal of Psychiatry 163, 433-440.

Kendler KS (2008). Explanatory models for psychiatric illness. American Journal of Psychiatry 165, 695-702.

Kendler KS (2012). The dappled nature of causes of psychiatric illness: replacing the organic-functional/ hardware-software dichotomy with empirically based pluralism. Molecular Psychiatry 17, 377-388.
Mellor CS (1970). First rank symptoms of schizophrenia. I. The frequency in schizophrenics on admission to hospital. II. Differences between individual first rank symptoms. British Journal of Psychiatry 117, 15-23.

Miall RC, Weir DJ, Wolpert DM, Stein JF (1993). Is the cerebellum a Smith predictor? Journal of Motive Behavior 25, 203-216.

Mishara AL (2010). Klaus Conrad (1905-1961): delusional mood, psychosis, and beginning schizophrenia. Schizophrenia Bulletin 36, 9-13.

Nagel T (1979). Mortal Questions. Cambridge University Press: New York.

Robbins TW, Gillan CM, Smith DG, de Witt S, Ersche KD (2012). Neurocognitive endophenotypes of impulsivity and compulsivity: towards dimensional psychiatry. Trends in Cognitive Sciences 16, 81-91.

Schneider K (1959). Clinical Psychopathology. Grune \& Stratton: New York.

Wikipedia contributors (2010). Wilhelm Dilthey (http:// en.wikipedia.org/w/index.php?title=Wilhelm Dilthey\&oldid=369814642). Accessed 12 July 2010. 